\title{
Temporary Transcutaneous Pacing in a Low Birth Weight Preterm Neonate with Congenital Complete Atrioventri- cular Block: A Case Report
}

Na Hyun Lee, M.D., So Young Shin, M.D., Ji Hyun Park, M.D., Jae Hyun Park, M.D., Chun Soo Kim, M.D., and Hee Joung Choi, M.D.

Department of Pediatrics, Keimyung University School of Medicine, Daegu, Korea

\section{ABSTRACT}

Congenital complete atrioventricular (AV) block is a rare neonatal disease. It is a passively acquired immune-mediated injury of the conduction system, triggered by transplacental passage of maternal anti-SSA/Ro and anti-SSB/La antibodies. Management of premature infants with symptomatic complete AV block is challenging. If medical treatment with a $\beta$-adrenergic agonist and inotropic drugs is not effective, early cardiac pacing should be considered. Here we report a case of congenital complete AV block in a low birth weight, preterm neonate, who was successfully treated with temporary transcutaneous pacing immediately after birth. Temporary transcutaneous pacing may be an option for the emergent management of a low birth weight preterm neonate with congenital complete AV block prior to permanent pacemaker implantation.

Key Words: Congenital complete atrioventricular block, Cardiac pacing

\section{INTRODUCTION}

Congenital atrioventricular (AV) block is a rare neonatal disease with an overall prevalence of one in 15,000-20,000 live births ${ }^{1)}$. About $80-95 \%$ of neonates with isolated congenital AV block without structural heart disease are associated with neonatal lupus erythematosus. Congenital AV block is a passively acquired autoimmune disease, in which an inflammatory response is triggered by the transplacental passage of maternal antiSSA/Ro and anti-SSB/La antibodies ${ }^{2}$. This immune-mediated inflammation results in myocardial tissue injury, fibrosis, and scarring of the conduction system ${ }^{1,2)}$. The conduction disturbance in the fetus, including first, second, and third-degree AV block, is progressive. However, complete AV block is usually irreversible, and most patients need pacemaker implantation ${ }^{3)}$.

Here we present the case of a low birth weight, preterm neonate with congenital complete AV block that was successfully managed with temporary transcutaneous pacing
Received: 12 August 2016

Revised: 29 September 2016

Accepted: 3 October 2016

Correspondence to: Hee Joung Choi

Department of Pediatrics, Keimyung

University School of Medicine

56 Dalseong-ro, Jung-gu, Daegu

41931, Korea

Tel: +82-53-250-7524

Fax: +82-53-250-7783

E-mail: joung756@dsmc.or.kr

Copyright(c)

By Korean Society of Neonatology.

All right reserved.

This is an Open-Access article distributed under the terms of the Creative Commons Attribution Non-Commercial License (http://creativecommons.org/licenses/ by-nc/4.0), which permits unrestricted non-commercial use, distribution, and reproduction in any medium, provided the original work is properly cited. 
immediately after birth.

\section{CASE REPORT}

A 33-year-old, nulli-paraous (gravida 1, para 0) mother was referred from a primary maternity hospital at $31^{+6}$ weeks of gestation due to fetal arrhythmia with bradycardia of 60 beats per minute (BPM). She had no autoimmune disease before pregnancy, although a malar rash was observed on her face upon admission. Laboratory examination revealed anemia, proteinuria, hematuria, elevated levels of blood urea nitrogen (BUN) and creatinine, and hypocomplementemia. The patient tested positive for antinuclear antibodies (ANA, 1:1280), anti-ds DNA antibodies (1:320), and anti-SSA/Ro antibodies (>200 U/ ml) but negative for anti-SSB/La antibodies. She was diagnosed with systemic lupus erythematosus with lupus nephritis.

A fetal echocardiogram showed discordant atrioventricular contraction with a ventricular rate of 55-60 BPM and fetal distress with heart failure. Consequently, an emergency cesarean section was performed. The male baby's Apgar scores at minutes 1 and 5 were 4 and 5 , while his birth weight was $1,710 \mathrm{~g}$ at $31^{+6}$ weeks of gestational age. There were no clinical signs of hydrops fetalis. He showed poor crying, cyanosis, grunting, and chest retraction. A chest radiograph revealed a total white-out pattern of respiratory distress syndrome (Figure 1A), and he was therefore intubated, treated with surfactant, and provided with ventilator assistance. His heart rate ranged between 50 and $60 \mathrm{bpm}, \mathrm{O}_{2}$ saturation was $93 \%$ by pulse oximetry, and blood pressure (BP) was undetected by automatic manometer. Continuous intravenous infusion of
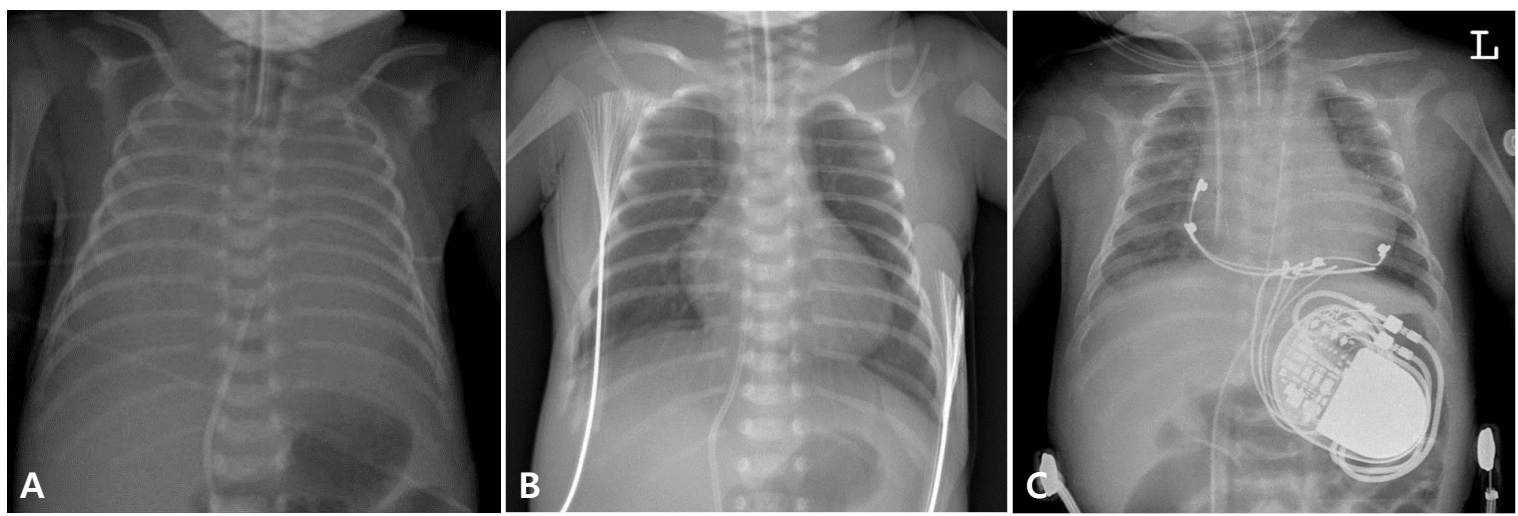

Figure 1. The patient's X-ray findings. (A) At birth, chest radiograph revealed a total white-out pattern in both lungs. (B) Chest radiograph with transcutaneous pacing at 2 days after birth. (C) Chest and abdominal radiograph after permanent pacemaker implantation at 99 days after birth.

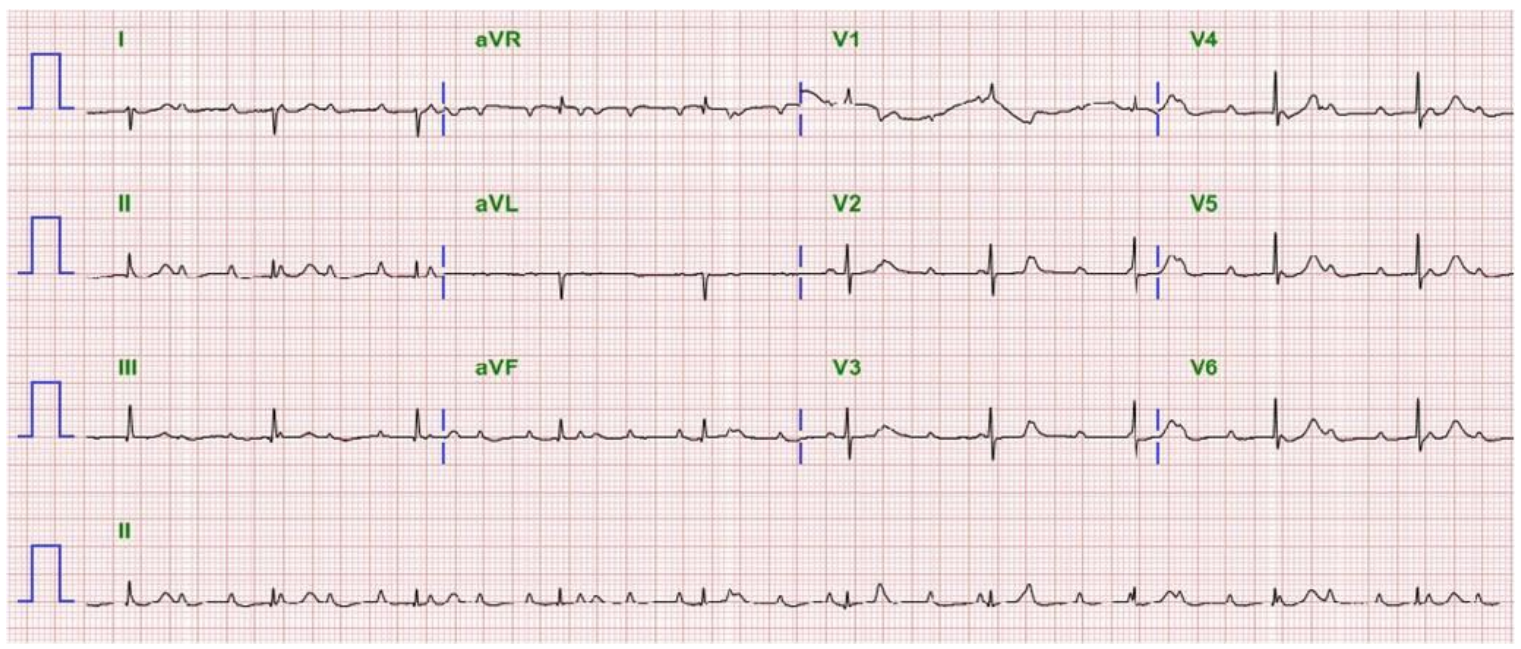

Figure 2. At birth, electrocardiography (ECG) showed complete atrioventricular block with an atrial rate of 167 BPM and a ventricular rate of $60 \mathrm{BPM}$. Abbreviation: BPM, beats per minute. 
inotropic drugs (dopamine and dobutamine, up to $20 \mu \mathrm{g} / \mathrm{kg} /$ $\mathrm{min}$ ) and isoproterenol (up to $0.2 \mu \mathrm{g} / \mathrm{kg} / \mathrm{min}$ ) was administered to treat hypotension and bradycardia. Electrocardiography (ECG) indicated complete AV block with an atrial rate of 167 BPM and a ventricular rate of 60 BPM (Figure 2). Two-dimensional (2D) echocardiography revealed decreased left ventricular contractility, atrial septal defect, patent ductus arteriosus, and grade III tricuspid regurgitation.

Intravenous drug treatment did not improve either the bradycardia or the hypotension. Temporary transcutaneous pacing (LIFEPAK 20 Defibrillator, Physio Control, USA) was applied with a ventricular rate of $100 \mathrm{BPM}$ and a pacing threshold of 30 $\mathrm{mA}$ five hours after birth. Adult external pacing electrodes were tailored to approximately $3 \times 3 \mathrm{~cm}$ in size and attached to the right anterior upper and left lower chest (Figures 1B, 3A). Immediately after transcutaneous pacing, his BP increased to $58 / 30 \mathrm{mmHg}$. There were two, $0.5 \mathrm{~cm}$-sized, oval-shaped contact burns (deep second - third degree) on the skin at the upper margin of the pad attachment site at 3 hours after transcutaneous pacing (Figure 3B). We therefore covered the margin of the pad with Duoderm ${ }^{\circledR}$ and applied an ointment on skin. Temporary transcutaneous pacing was continued without aggravation of the burn wounds, and was ceased three days after birth, as vital signs were stable with a mean BP of 40-50 mmHg and heart rate of 65-75 BPM. The burn wounds were treated with an ointment and foam dressing after consultation with plastic surgeons and healed one month after birth without scarring.

The patient tested positive for ANA and negative for both anti-

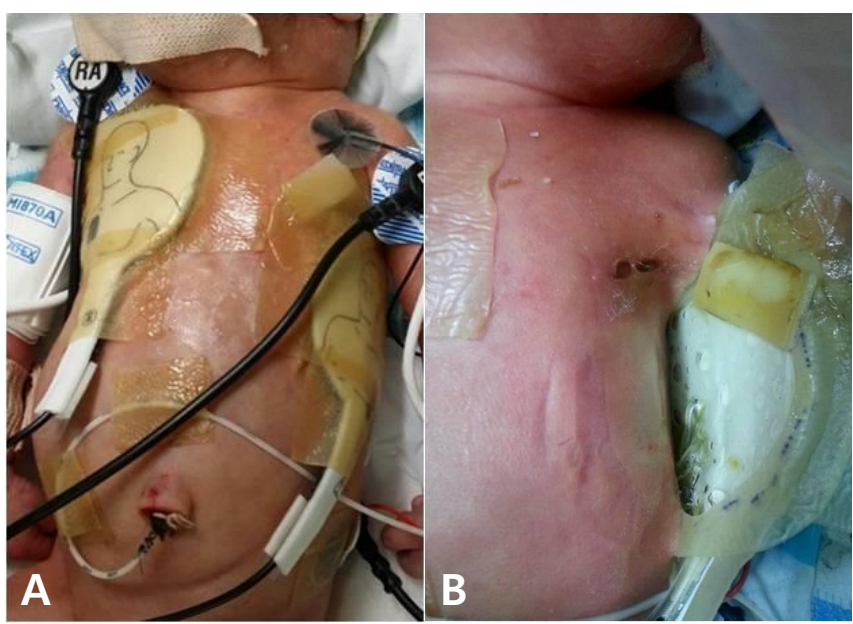

Figure 3. Appearance of this patient. (A) Transcutaneous pacing electrodes attached on the right anterior upper chest and left lower chest. (B) Two, oval-shaped contact burns at the upper margin of the pacing pad.
SSA/Ro antibodies and anti-phospholipid antibodies. Intravenous immunoglobulin $(1 \mathrm{~g} / \mathrm{kg})$ was administered twice. We observed his vital signs with use of isoproterenol and waited for the optimal timing for pacemaker implantation, preferably as late as possible. At 89 days after birth, we chose to implant a permanent pacemaker as gradual dilatation of the left ventricle was observed on 2D echocardiography. The patient was transferred to another tertiary hospital wherein a permanent epicardial pacemaker was successfully inserted at 99 days after birth with a recorded body weight of 1,980 $\mathrm{g}$ (Figure 1C). Presently, he is 20 months old in corrected age and doing well with proper weight gain. Good cardiac contractility has been maintained with no further dilatation of the left ventricle.

\section{DISCUSSION}

Congenital AV block is the most frequent and serious complication of neonatal lupus erythematosus (NLE) with an incidence of $15-30 \%$ in infants with NLE. Maternal anti-SSA/Ro and anti-SSB/La antibodies are transferred to the fetus during gestational weeks 16-24. The immune-mediated inflammation affects heart development, especially that of the conduction system $^{1,2)}$. Clinical signs of congenital AV block are generally detected during 18-24 weeks of gestation as a first or seconddegree AV block; however, most cases progress to a third, complete AV block ${ }^{2}$. These progressive conduction abnormalities can be prevented if detected and treated early, and it is recommended that antibody-positive mothers undergo close fetal echocardiographic surveillance in the early second trimester ${ }^{4}$. Nevertheless, most mothers are asymptomatic despite the presence of autoantibodies and are diagnosed with autoimmune diseases, such as systemic lupus erythematosus after diagnosis of congenital AV block or hydrops fetalis in the fetus or newborn ${ }^{1)}$. Additionally, fetal exposure to high titers of anti-SSA/Ro antibodies is strongly correlated with cardiac damage. Jaeggi et al. reported that complete AV block occurred with anti-SSA/Ro levels $>100 \mathrm{U} / \mathrm{mL}$, but never occurred in cases with levels $<50$ $\mathrm{U} / \mathrm{mL}^{5}$. In the present case, there was no clinical evidence of maternal autoimmune disease either before or during preg. nancy, and close antenatal observation or management was not performed prior to delivery. The maternal titer of anti-SSA/ Ro was greater than $200 \mathrm{U} / \mathrm{mL}$, and the infant had complete AV block. 
The clinical features of neonates with congenital complete AV block range from absence of any symptoms to severe heart failure and hydrops fetalis. Myocarditis, pericardial effusions, endocardial fibroelastosis, and dilated cardiomyopathy can all occur simultaneously with congenital AV block, with the most severe in utero complication being fetal death due to low cardiac output $^{6}$. Indeed, the reported perinatal mortality rate is approximately $20-30 \%^{2)}$. There is a particularly poor prognosis for cases with a heart rate below 50-55 BPM, fetal hydrops, endocardial fibroelastosis, impaired left ventricular function, or those at less than 20 gestational weeks ${ }^{3,6,7)}$.

A clear protocol for the perinatal management of congenital AV block has not yet been established. However, transplacental steroids and sympathomimetic ( $\beta$-adrenergic agonist) therapies may reduce myocardial inflammation with first and seconddegree AV block ${ }^{2,8)}$. Ruffatti et al. showed that there is a possibility of reverting or blocking the progression of heart damage by combining antenatal therapy with plasmapheresis, intravenous immunoglobulin (IVIG), and betamethasone (fluorinated steroids). This treatment is continued into the postnatal period with IVIG until maternal autoantibodies become undetectable. This combination therapy may assist in the complete regression of first and second-degree AV block; however, it can only prevent cases of third-degree AV block from progressing to heart failure ${ }^{4)}$. Thus, perinatal management should be initiated as soon as possible.

If a fetus with third-degree, complete AV block shows fetal distress or deteriorating cardiac performance, delivery should be considered, which can be contributor to preterm birth. The management of premature infants with symptomatic complete AV block is challenging, and includes drug treatment, temporary cardiac pacing, and permanent pacemaker implantation ${ }^{1}$. Drugs such as isoproterenol ( $\beta$-adrenergic agonist), epinephrine, dopamine, and atropine are used to increase the infant's heart rate if it is below $60 \mathrm{BPM}$; however, these drugs are rarely successful. Immediate pacing should therefore be considered in most cases, even as a first strategy before commencing a drug trial $^{9)}$.

Indications for permanent pacemaker implantation with congenital complete AV block include patients with (1) symptomatic bradycardia, congestive heart failure, or low cardiac ourput; (2) a resting heart rate $<55 \mathrm{BPM}$; (3) left ventricular dilatation or dysfunction; (4) pauses $>3$ seconds during Holter monitoring; (5) a broad ventricular escape rhythm (QRS duration $>120$ $\mathrm{ms})$; or (6) a prolonged QT interval ${ }^{1,10)}$. The critical decision is when to initiate pacing therapy, as the majority of patients will require it for life. Recent case reports have demonstrated that a permanent epicardial pacemaker can be successfully implanted immediately after birth in very low birth weight infants with congenital complete AV block ${ }^{11-13)}$. However, early implantation in very young and small patients has a greater risk of poor cosmetic results with multiple scars, skin erosion and infection, lead extraction or fracture, generator migration, and re-implantation ${ }^{14,15)}$. Moreover, in preterm and low birth weight neonates, patients are very unstable in terms of undergoing such an invasive surgical procedure ${ }^{9,14)}$.

Temporary transcutaneous pacing could be one option for emergent management of thise condition. Temporary transcutaneous pacing has been broadly used in older children and adults to manage acute bradycardia, but the large size of pacing electrodes limits its use in neonates and infants. In 1999, Rein et al. reported two cases of complete AV block newborns treated with external noninvasive pacemakers immediately after birth ${ }^{9)}$. In Korea, a case of transient complete AV block was reported in a neonate with congenital myotonic dystrophy where temporary transcutaneous pacing was applied 4 hours after birth ${ }^{16)}$. When compared with temporary epicardial pacing, the advantages of this technique are that it is non-invasive, relatively simple to use, and rapidly available after delivery ${ }^{15)}$. Thermal injury caused by electrolytic reaction occasionally occurs at the site of electrode attachment, and children and infants are particularly susceptible. Moreover, physicians have had to tailor large adultsized external pacing electrodes for small preterm neonates, resulting in an increased risk of burns caused by the unprotected surfaces of the pacing electrodes. In order to prevent thermal injury, it is necessary to closely monitor electrode contact sites and use the lowest effective current ${ }^{9,15,16)}$. As in our case, it may be helpful to wrap the margin of the tailored pacing electrodes with an insulating material to prevent thermal injury. In this case, we successfully stabilized a low birth weight, preterm neonate with congenital complete AV block with temporary transcutaneous pacing during a hemodynamically unstable period immediately after birth. We were further able to successfully discontinue pacing therapy after 3 days without bradycardia or hypotension. Although there was thermal injury on the left chest, this healed completely and no major complications were experienced.

In conclusion, temporary transcutaneous pacing is a treatment option for a low birth weight, premature neonate with congenital 
complete AV block immediately after birth.

\section{REFERENCES}

1) Bordachar P, Zachary W, Ploux S, Labrousse L, Haissaguerre M, Thambo JB. Pathophysiology, clinical course, and management of congenital complete atrioventricular block. Heart Rhythm 2013;10:760-6.

2) Hutter D, Silverman ED, Jaeggi ET. The benefits of transplacental treatment of isolated congenital complete heart block associated with maternal anti-Ro/SSA antibodies: a review. Scand J Immunol 2010;72:235-41.

3) Breur JM, Kapusta L, Stoutenbeek P, Visser GH, van den Berg P, Meijboom EJ. Isolated congenital atrioventricular block diagnosed in utero: natural history and outcome. J Matern Fetal Neonatal Med 2008;21:469-76.

4) Ruffatti A, Marson P, Svaluto-Moreolo G, Marozio L, Tibaldi M, Favaro M, et al. A combination therapy protocol of plasmapheresis, intravenous immunoglobulins and betamethasone to treat anti-Ro/La-related congenital atrioventricular block. A case series and review of the literature. Autoimmun Rev 2013;12:768-73.

5) Jaeggi E, Laskin C, Hamilton R, Kingdom J, Silverman E. The importance of the level of maternal anti-Ro/SSA antibodies as a prognostic marker of the development of cardiac neonatal lupus erythematosus a prospective study of 186 antibodyexposed fetuses and infants. J Am Coll Cardiol 2010;55:277884.

6) Hon KL, Leung AK. Neonatal lupus erythematosus. Autoimmune Dis 2012;2012:301274.

7) Eliasson H, Sonesson SE, Sharland G, Granath F, Simpson JM, Carvalho JS, et al. Isolated atrioventricular block in the fetus: a retrospective, multinational, multicenter study of 175 patients. Circulation 2011;124:1919-26.

8) Jaeggi ET, Fouron JC, Silverman ED, Ryan G, Smallhorn J, Hornberger LK. Transplacental fetal treatment improves the outcome of prenatally diagnosed complete atrioventricular block without structural heart disease. Circulation 2004;110: 1542-8.

9) Rein AJ, Cohen E, Weiss A, Marks KA, Peleg O, Nir A. Noninvasive external pacing in the newborn. Pediatr Cardiol 1999; 20:290-2.

10) Epstein AE, DiMarco JP, Ellenbogen KA, Estes NA 3rd, Freedman RA, Gettes LS, et al. 2012 ACCF/AHA/HRS focused update incorporated into the ACCF/AHA/HRS 2008 guidelines for device-based therapy of cardiac rhythm abnormalities: a report of the American College of Cardiology Foundation/American Heart Association Task Force on Practice Guidelines and the Heart Rhythm Society. J Am Coll Cardiol 2013;61:e6-75.

11) Donofrio MT, Gullquist SD, Mehta ID, Moskowitz WB. Congenital complete heart block: fetal management protocol, review of the literature, and report of the smallest successful pacemaker implantation. J Perinatol 2004;24:112-7.

12) Welch EM, Hannan RL, DeCampli WM, Rossi AF, Fishberger SB, Zabinsky JA, et al. Urgent permanent pacemaker implan tation in critically ill preterm infants. Ann Thorac Surg 2010; 90:274-6.

13) Baek SH, Ahn SY, Lee MS, Han YM, Sung SI, Yoo HS, et al. A case of pacemaker implantation in premature newborn with congenital complete atrioventricular block. J Korean Soc Neonatol 2012;19:275-9.

14) Shepard CW, Kochilas L, Vinocur JM, Bryant R, Harvey BA, Bradley S, et al. Surgical placement of permanent epicardial pacing systems in very low-birth weight premature neonates: a review of data from the eediatric cardiac care consortium (PCCC). World J Pediatr Congenit Heart Surg 2012;3:454-8.

15) Maginot KR, Mathewson JW, Bichell DP, Perry JC. Applications of pacing strategies in neonates and infants. Prog Pediatr Cardiol 2000;11:65-75.

16) Kim HN, Cho YK, Cho JH, Yang EM, Song ES, Choi YY. Transient complete atrioventricular block in a preterm neo nate with congenital myotonic dystrophy: case report. J Korean Med Sci 2014;29:879-83. 